\title{
Application of Fan Boundary Condition for Modelling Helicopter Rotors in Vertical Flight
}

\author{
Anna A. Kostek ${ }^{1,2,3(\bowtie)}$, Katarzyna Surmacz ${ }^{1}$, Michał Rajek ${ }^{1}$, \\ and Tomasz Goetzendorf-Grabowski ${ }^{2}$ \\ 1 Lukasiewicz Research Network - Institute of Aviation, Krakowska Av. 110/114, \\ 02-256 Warsaw, Poland \\ anna.kostek@dlr.de \\ 2 Warsaw University of Technology, Warsaw, Poland \\ 3 Now at German Aerospace Center (DLR), Göttingen, Germany
}

\begin{abstract}
The fan boundary condition in Ansys Fluent was applied to a helicopter rotor in vertical flight using a Robinson R22 geometry. This simplified boundary condition, which is based on a pressure jump over an actuator disk, offers considerable advantages in speed and stability compared to methods using a blade element theory. It was shown that the identification of different rotor working states and preparation of the induced velocity curve is possible by applying the analyzed rotor model. Particular attention was given to the prediction of the vortex ring state - a phenomenon that cannot be described using the momentum theory and which poses a significant threat during helicopter flight. The outcomes were comparable to those of experimental visualizations and simulations performed using the more computationally expensive Virtual Blade Model, thereby proving the viability of the fan boundary condition to model the main rotors.
\end{abstract}

Keywords: Fan boundary condition - Virtual Blade Model • Computational models for main rotors - Helicopter vertical flight • Robinson R22 - Rotor working states $\cdot$ Vortex ring state

\section{Introduction}

Vertical flight of a helicopter involves its rotor operating in various flow conditions, most of which can be satisfactorily described by means of the momentum theory. However, some of them, like the vortex ring state, are accompanied by particularly complex aerodynamic phenomena, for which the simplified theory is not applicable. At the same time a thorough flow analysis under such conditions is extremely important because of the risks involved. CFD software, alongside with tunnel experiments, provides a tool to investigate complicated vortex structures. However, the choice of computational methods, including modelling of the main rotor, usually has to be a compromise between numerical costs and

(C) The Author(s), under exclusive license to Springer Nature Switzerland AG 2021

A. Dillmann et al. (Eds.): STAB/DGLR Symposium 2020, NNFM 151, pp. 355-364, 2021.

https://doi.org/10.1007/978-3-030-79561-0_34 
the accuracy level of the results. This means, depending on the purpose of the research, appropriate simplifications should be applied.

Choosing the fan boundary condition available in the Ansys Fluent software as a model of a main rotor significantly reduces preparation and calculation time, however, its application is limited only to cases considering the axial flow through the rotor, possibly with a low lateral velocity. The question is, whether, despite of its simplified assumptions, the fan boundary condition is capable of reproducing the variety of phenomena associated with helicopter vertical flight.

\section{Methodology}

The fan is one of the boundary conditions of the internal surface type, which introduce step changes in flow parameters. The rotor model in this method is presented as an infinitely thin surface of a pressure jump, which allows for an average effect of blade rotation. In the presented analysis a uniform pressure jump across the disk was assumed (Fig. 1). According to [2] wake swirl can be considered in the calculations after determining tangential and radial velocity distribution along the rotor radius (Fig. 2) as polynomial functions (1) and (2) respectively:

$$
u_{\theta}=\sum_{n=-1}^{N} f_{n} r^{n}
$$

$$
u_{r}=\sum_{n=-1}^{N} g_{n} r^{n}
$$

where $f_{n}, g_{n}$ are the function coefficients, $r$ is the radial distance from the hub and $-1 \leq \mathrm{N} \leq 6$.

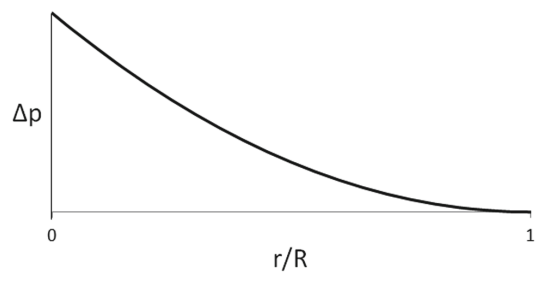

Fig. 1. Uniform pressure jump distribution along the disk radius

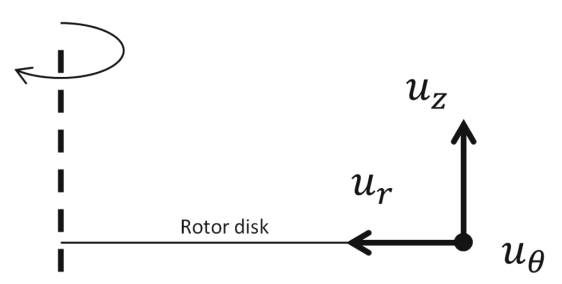

Fig. 2. Velocity components in the applied coordinate system

The Virtual Blade Model (VBM) is a more complex approach based on the blade element theory [13]. In this case the rotor is presented as a single layer of elements (here structural, as shown later on Fig. 5b). The required input includes detailed information on the geometric and mass properties of rotor blades, along with aerodynamic characteristics of profiles, and rotor parameters, e.g. its orientation, rotational speed, collective and cyclic angles. On this basis 
the time-averaged effects of blades rotation can be simulated using individual cells of the rotor disk as momentum sources, which are then included in NavierStokes equations as a vector of external forces.

\subsection{Numerical Setup}

A model of the Robinson R22 helicopter was reconstructed by means of NX Unigraphics software [12] on the basis of [10] and placed in the middle of a cube-shaped domain with a side length ten times the rotor diameter (Fig. 3). ANSYS Icem software [1] was used to prepare an unstructured tetrahedral grid with layers of prism elements covering the boundary layer around the fuselage (Fig. 4).

Simulations concerned the range of flight velocities $V$ up to $8 \mathrm{~m} / \mathrm{s}$ for the axial climb and $17 \mathrm{~m} / \mathrm{s}$ for the axial descent using grids of 1.2 and 3.2 million elements (Mesh I, Mesh II) in a steady mode and additionally with a transient flow for Mesh II. A single-equation Spalart-Allmaras turbulence model was chosen as sufficient for the needs of analysis of large-scale turbulent structures characteristic for vertical flight of the helicopter. The fan boundary condition was set on the main and tail rotor disk surfaces with the constant values of the pressure jump of $107 \mathrm{~Pa}$ and $340 \mathrm{~Pa}$ respectively, without considering swirl.
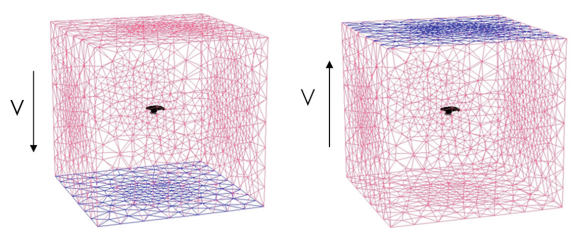

Fig. 3. Computational domain for descent and climb; pink: pressure far-field, blue: pres-

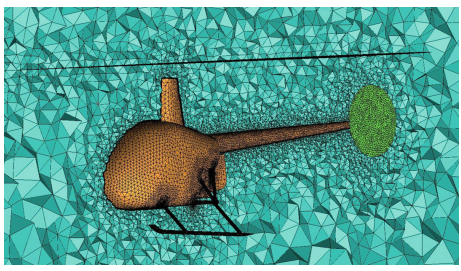

Fig. 4. Grid setup sure outlet

Wake swirl modelling available in the fan boundary condition was tested for a hover case on the basis of comparison with the VBM results using the same grid setup (Mesh I). The only obvious differences in the grids were related to the rotor model (Fig. 5). In the end, for comparison purposes, the tail rotor operation was not taken into account and a simplified helicopter model with no skids was used.

A function defining the tangential component of the wake swirl velocity in hover was modelled on the basis of two approaches described in [7] - actuator disk vortex theory and momentum theory extended with swirl. The first one assumes for a uniformly loaded rotor that a tangential component of velocity $u_{\theta}$ added to the flow equals the strength of the vorticity $\Gamma$ : 


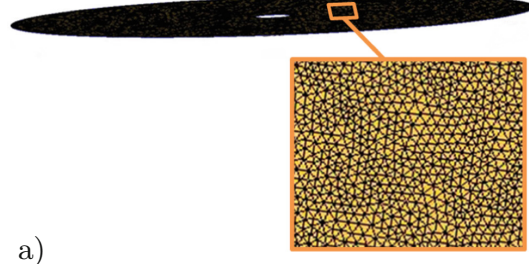

a)

Fig. 5. Main rotor representations; a) fan boundary condition b) Virtual Blade Model

$$
u_{\theta}=\frac{\Gamma}{2 \pi r}=\frac{T}{\rho A \omega r},
$$

where $\mathrm{T}$ is the thrust force, $\mathrm{A}$ the rotor disk area and $\omega$ the rotational speed of the rotor. The second equation takes into account the wake swirl arising from induced and profile drag (6), where $v_{h}$ is the induced velocity in hover and $c_{l}, c_{d}$ stand for the lift and drag coefficient respectively.

$$
u_{\theta}=v_{h}\left[\frac{2 v_{h} \omega r}{(\omega r)^{2}+v_{h}^{2}}+2 \frac{c_{d}}{c_{l}}\right],
$$

Functions $u_{\theta 2}$ and $u_{\theta 3}$, displayed on Fig. 6 , are examples of approximations of the tangential velocity distribution according to (3) and (4). The increase in tangential velocity caused by turbulence at the edge of the rotor disk has been considered for the function $u_{\theta 4}$, for which a velocity peak up to the value of $v_{h}$ was added on the basis of experimental results [3]. For the radial velocity distribution an approximating polynomial function $u_{r 2}$ was suggested based on an analytical model [5] (Fig. 7), along with a simple, linear function $u_{r 1}$ with the maximum value equal $v_{h}$ at its end.

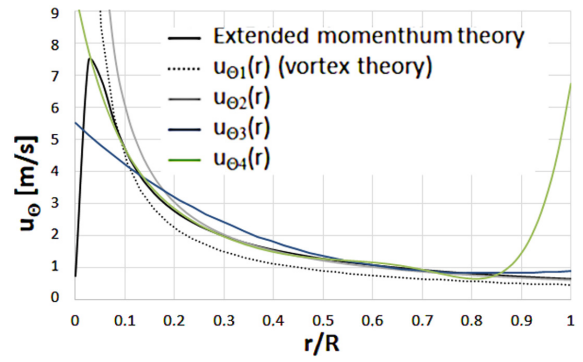

Fig. 6. Functions approximating tangential velocity distribution

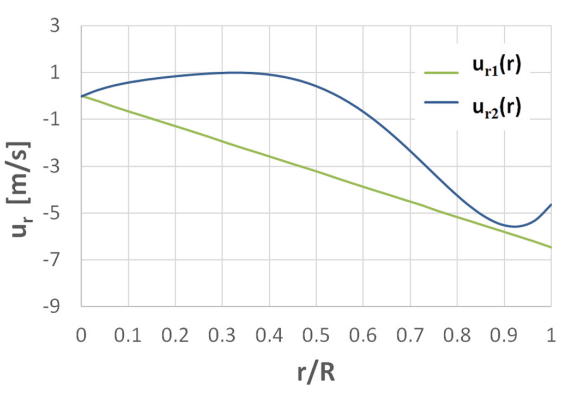

Fig. 7. Functions approximating radial velocity distribution 


\section{Results}

\subsection{Identification of Rotor Working States}

Figure 8 presents changes in the induced velocity $v$ depending on the vertical flight velocity $V$ obtained from numerical simulations. The effect of these changes is visible on Fig. 9 showing induced power as a function of $V$ according to the momenthum theory (5). The average values of the induced velocity $v$ were calculated from the resulting mass flow rate through the rotor using the equation (6), where $\rho$ is the air density and $A$ is the disk area:

$$
\bar{P}=\bar{v}+\bar{V}
$$

$$
\dot{m}=\rho(v+V) A
$$

Labels $\bar{v}$ and $\bar{V}$ correspond to the values of velocities nondimensionalised by the induced velocity in hover. Similarly, $\bar{P}$ is the power value divided by the power value in hover.

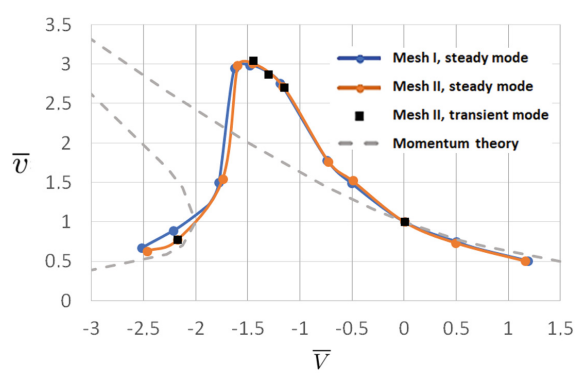

Fig. 8. Induced velocity curve

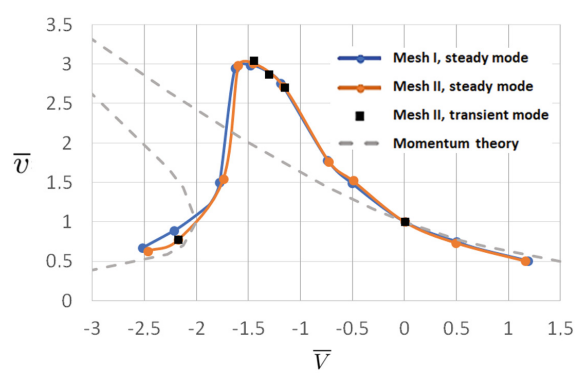

Fig. 9. Power curve

The presented plots show that refinement of the mesh did not influence much the results, so further calculations from Sect. 3.2 were conducted using Mesh I. As expected, for the range of velocities corresponding to the normal working state $(\bar{V} \geq 0)$ and windmill brake state $(\bar{V} \leq-2)$ obtained results are consistent with the momentum theory [9]. The highest calculated value of the induced velocity is as much as three times higher than the value of the induced velocity in hover and was reached around $\bar{V}=-1.5$. Similar values were also observable during experiments $[4,16]$. The resulting loss in induced power causing increased power consumption (Fig. 9) characterizes flight in the vortex ring state. The steep decline in $v$ for higher flight velocities followed by a change to negative power values indicates entering the autorotative flight regime, typical for the turbulent wake state.

The presence of particular flow states in the numerical simulations using the fan boundary condition can be verified closely when compared with the results 
from experiments $[11,14]$. Smoke visualizations from the wind tunnel test of a descending helicopter model showed apparent similarities for the corresponding nondimensionalised flight velocity values as presented on Fig. 10.

For low descent speeds, the direction of flow just underneath the rotor is determined by the mass of induced air, resulting in a lack of noticeable circulation on the smoke visualization (Fig. 10a). However, as the descent velocity increases, the coming mass of air moves closer to the rotor disk until it reaches the rotor around $\bar{V}=1$. From this point on, the increase in flow velocity causes a clear recirculation of air around the rotor, which indicates a fully developed vortex ring state (Fig. 10b). The largest diameter of the vortex with the length of approximately the rotor diameter was observed around the flow velocity $\bar{V}=-1.2$ for both analyzes. The process of the development and vanishing of the vortex ring state was observed in detail by means of PIV (Particle Image Velocimetry) in $[14,15]$. As a result of the speed increase, the vortex ring starts to shrink and concentrate closer to the rotor disk, while at the same time cores of vortices move gradually over the rotor surface. Similar changes in the vortex ring geometry were obtained from the numerical simulations (Fig. 11).

As the velocity increases further, recirculation weakens and thus the net-flow through the rotor disk is nearly zero $(\bar{V}=-1.7)$. Consequently the area of turbulence characteristic for the turbulent wake state appears above the rotor plane (Fig. 10c). For high descent speeds, as the turbulence fades, the flow is again well-defined and does not change significantly with the further increase in velocity. As the flow velocity notably exceeds the induced velocity, smoke reaches far behind the rotor disk. The flow is now directed upwards being fully determined by the mass of coming air (Fig. 10d). The presented visualization reflects the description of the windmill brake state from the momentum theory.

a)

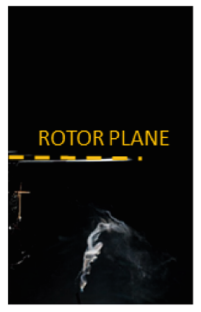

c)

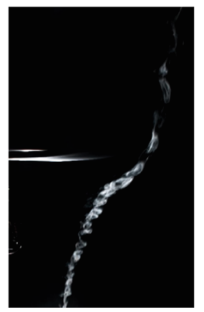

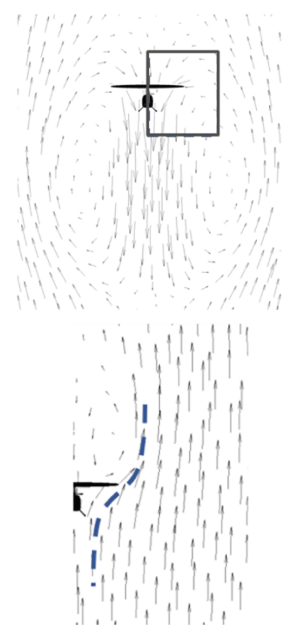

b)
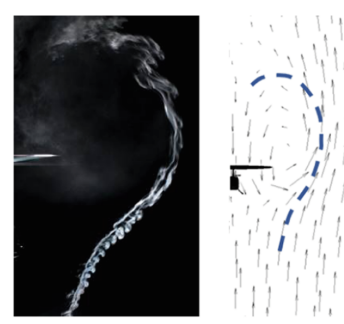

d)
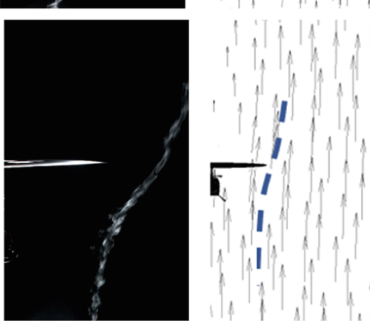

Fig. 10. Comparison between smoke visualizations and numerical analysis of flow around a descending helicopter rotor; a) $\bar{V}=-0.7$ b) $\bar{V}=-1.2$ c) $\bar{V}=-1.7$ d) $\bar{V}=-2.8$ 

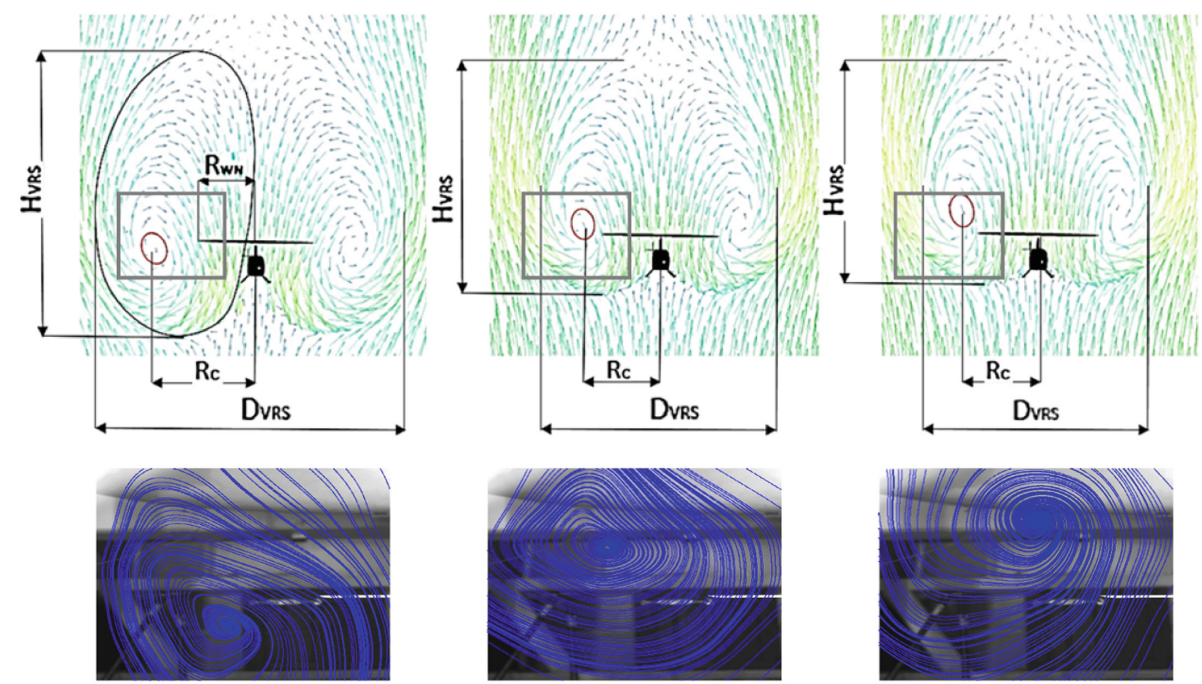

Fig. 11. Change in geometry of vortex ring with increasing descent rate for $\bar{V}=-1.2$, $\bar{V}=-1.3, \bar{V}=-1.5$ compared with PIV visualizations, symbols based on [6]

\subsection{Wake Swirl in Hover}

The obtained distributions of the tangential velocity from approximating functions (Fig. 6) clearly show better agreement with the results produced by means of the VBM than primary settings of the fan (Fig. 12).

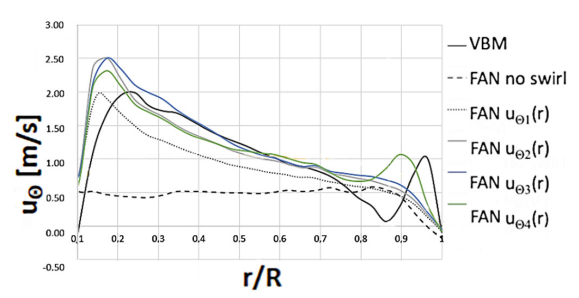

Fig. 12. Tangential velocity distribution obtained from numerical calculations (azimuth $90^{\circ}$ )

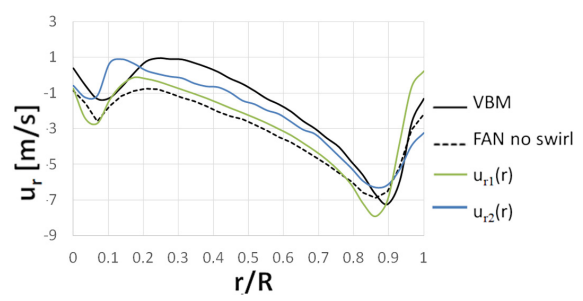

Fig. 13. Radial velocity distribution obtained from numerical calculations (azimuth $90^{\circ}$ )

In order to obtain physical results when changing swirl parameters, it is important to define the distribution of tangential and radial velocity components in parallel. After applying function $u_{r 2}$ the radial velocity distribution was in better accordance with the VBM results than a primary model without swirl (Fig. 13). However, it also resulted in an incorrect axial velocity distribution, as presented on Fig. 14. More adequate induced velocity values were reached by modelling with a simplified function $u_{r 1}$. 


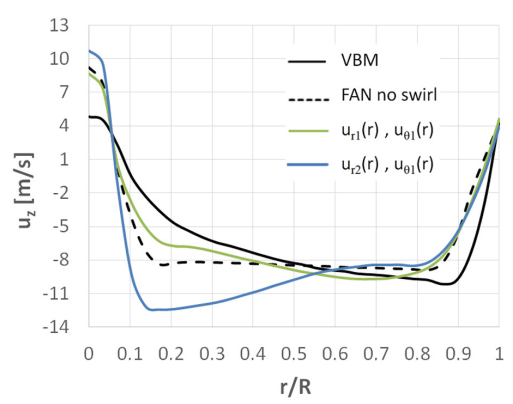

Fig. 14. Axial velocity distribution obtained after adding swirl (azimuth $\left.90^{\circ}\right)$

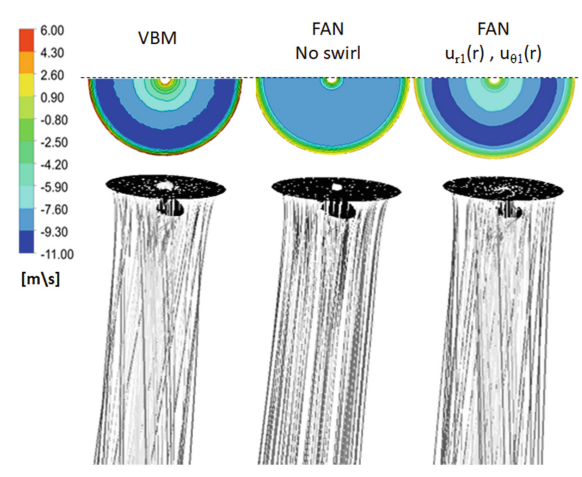

Fig. 15. Comparison of axial velocity contours on the rotor surface and change in wake swirl

\section{Discussion and Conclusions}

The conducted simulations allow for the identification of particular flow states with the range of their occurrence corresponding to the experimental results. This proves the validity of using the fan boundary condition as a model of a helicopter's main rotor for the purpose of vertical flight simulations. Figure 16 presenting the induced velocity curve additionally includes straight lines, which define theoretical limits of rotor working states during helicopter descent. Presented areas correspond to those obtained from numerical simulations.

Additionally, the application of basic settings of the fan boundary condition enables to observe stages of development of a vortex ring state, which is indescribable on the basis of theoretical approach and thus represents a particularly interesting point of analysis. Results obtained in this way provide the ground for the investigation of threats related to the above phenomenon and the development of escape techniques and prevention methods. However, it should be emphasized that the flow through the rotor in this condition is highly unsteady both in space and time, which means that simulations based on the fan boundary condition offer only a simplified, averaged presentation of the described state. The experiment [14] proved that generated vortex structures periodically expand until they are abruptly detached from the rotor blade. The resulting chaotic nature of the flow leads to fluctuations of the thrust force and high vibrations. As shown on Fig. 8 transient calculations did not significantly change the results compared to less time-consuming stationary analysis. The assumption of a constant value of the pressure jump and consequently a constant thrust force in defining the parameters of the fan boundary condition is a significant limitation for showing non-stationary effects of the rotor flow, regardless of the adopted calculation mode. However, the fan boundary condition settings allow the application of a function describing the pressure jump value depending on the inflow velocity. The example of defining this relation for hover can be found in [8]. 


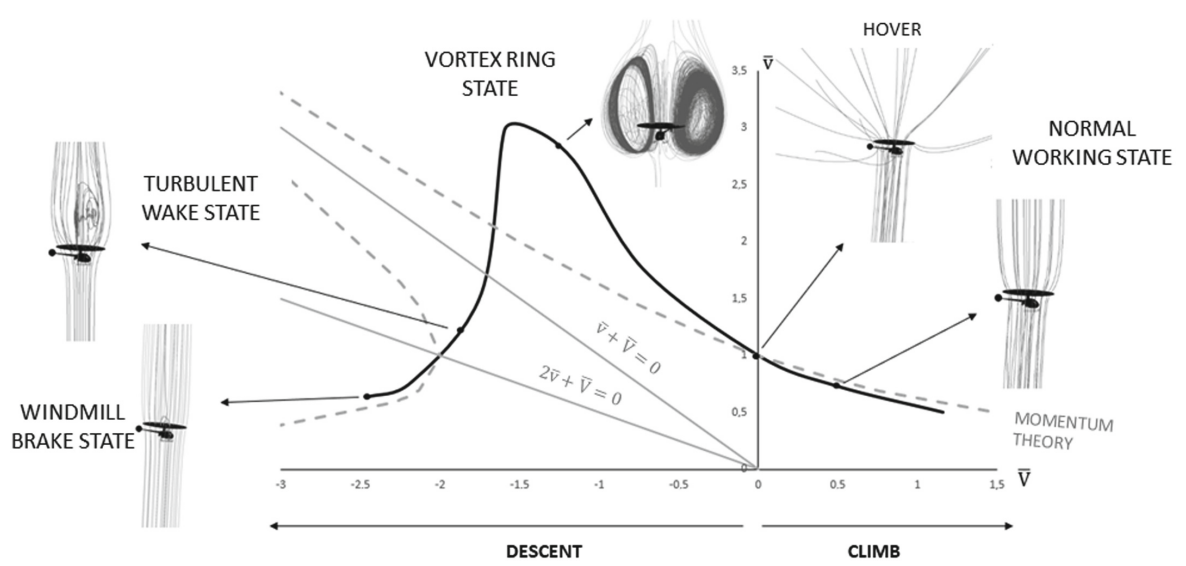

Fig. 16. Identification of main rotor working states based on simulations results

An advantage of the adopted model is its time-effectiveness, when it comes to both numerical costs and the process of preparing the rotor geometry. This is an essential advantage compared to the VBM that requires much input data, high quality grids and calculation times up to $20 \%$ longer. The simulation results indicate that the use of additional fan boundary condition settings for wake swirl modelling leads to outputs similar to the VBM model, but does not ensure complete consistency of results. Functions determining the distribution of velocity components along the rotor radius can be defined on the basis of theoretical equations and experimental results. It should be noted, however, that the suggested approximation functions are only applicable for hover, or can be modified for cases within the scope of momentum theory. To derive the above dependencies for the descent in the vortex ring state, if possible, would require much deeper analysis.

When defining the swirl settings, care should be taken with regard to changing associated parameters, as shown on Fig. 14. However, the adjusting of radial and tangential velocity components may allow for an indirect modelling of the axial velocity (Fig. 15). The lack of possibility of azimuthal correction of the introduced functions limits the application of the analyzed model of the main rotor to cases characterized by axisymmetrical flow through the rotor. For this reason, unlike the VBM, the fan boundary condition cannot be used for forward flight simulations. Another natural limitation resulting from adopting the main rotor model as a disk is the inability to simulate effects appearing on individual blades. One of them are changes in the geometry of tip vortices resulting from the increase in axial flight velocity, which were visible on smoke visualizations (Fig. 10). They present an additional feature characteristic for particular flow states, which, however cannot be detected with the use of both the fan boundary condition and the VBM. To summarize, the fan boundary condition adopted as a main rotor model enables to simulate the vertical flight of a helicopter with reduced calculation costs and the obtained results are a good starting point for more precise research. 


\section{References}

1. ANSYS Inc.: ANSYS ICEM CFD 14.5 User's Manual (2012)

2. ANSYS Inc.: ANSYS Fluent User's Guide (2013)

3. Boatwright, D.: Measurements of velocity components in the wake of a full-scale helicopter rotor in hover. Missisipi State Univeristy (1972)

4. Castles, W., Jr., Gray, R.B.: Empirical relation between induced velocity, thrust, and rate of descent of a helicopter rotor as determined by wind-tunnel tests on four model rotors. NACA-TN-2474 (1951)

5. Conway, J.T.: Exact actuator disk solutions for non-uniform heavy loading and slipstream contraction. J. Fluid Mech. 365(1), 235-267 (1998)

6. Grzegorczyk, K.: Wykorzystanie metod obliczeniowej mechaniki plynow do modelowania szczegolnego przypadku znizania smiglowca (VRS). Institute of Aviation, Warsaw (2010). [Eng.: Application of CFD methods for modelling a specific case of helicopter descent (VRS)]

7. Johnson, W.: Helicopter Theory. Dover Publications Inc., New York (1980)

8. Kostek, A.A.: Modelowanie wirnikow nosnych za pomoca warunku brzegowego fan na przykladzie smiglowca Robinson R22. M.Sc. thesis, Warsaw University of Technology (2019). [Eng.: Modelling of helicopter main rotors using the fan boundary conditon based on the example of the Robinson R22 helicopter]

9. Leishman, G.: Principles of Helicopter Aerodynamics. Cambridge University Press, Cambridge (2006)

10. Robinson Helicopter Company: R22 Maintenance Manual (2018)

11. Surmacz, K.: Dynamika zjawiska pierscienia wirowego na wirniku nosnym smiglowca. Ph.D. thesis, Air Force Institute of Technology, Warsaw (2015). [Eng.: Dynamics of the vortex ring state on the helicopter main rotor]

12. Siemens Documentation, NX 12 (2017)

13. Stalewski, W., Surmacz, K.: Investigations of the vortex ring state on a helicopter main rotor based on computational methodology using URANS solver. Lukasiewicz Research Network - Institute of Aviation, Warsaw (2019)

14. Stryczniewicz, W., Surmacz, K.: Badania eksperymentalne stanu pierscienia wirowego na wirniku nosnym smiglowca metoda anemometrii obrazowej (PIV). Institute of Aviation, Warsaw (2014). [Eng.: Experimental studies on the vortex ring state on helicopter main rotor using Particle Image Velocimetry (PIV)]

15. Surmacz, K., Ruchała, P., Stryczniewicz, W.: Wind tunnel tests of the development and demise of Vortex Ring State of the rotor. Institute of Aviation, Warsaw (2014)

16. Washizu, K., Azuma, A., Koo, J., Oka, T.: Experiments on a model helicopter rotor operating in the vortex ring state. J. Aircr. 3(3), 225-230 (1966) 